\title{
Detectors for the Beamline for Schools competition at CERN
}

\author{
Ina Carli ${ }^{*}{ }^{a, b}$ Candan Dozen, ${ }^{c}$ Saime Gurbuz, ${ }^{d}$ Markus Joos $\dagger,{ }^{a}$ Branislav Ristic $* a, e$ \\ Theodoros Vafeiadis, ${ }^{a}$ Oskar Wyszynski ${ }^{a}$ and Cenk Yildiz ${ }^{f}$ \\ a CERN, Switzerland \\ ${ }^{b}$ Charles University, Czech Republic \\ ${ }^{c}$ Cukurova University, Turkey \\ ${ }^{d}$ Bogazici University, Turkey \\ ${ }^{e}$ Lancaster University, UK \\ ${ }^{f}$ University of California Irvine, USA \\ E-mail: markus.joos@cern.ch, ina.carli@cern.ch, \\ branislav.risticecern.ch
}

CERN Beamline for Schools is an annual worldwide competition for high-school students. Teams of students are invited to propose an experiment with one of the secondary beams of the Proton Synchrotron and two winning experiments are performed each year by students with a help of CERN experts.

We will describe detectors available to students, with emphasis on design and performance of recently added Multi-Gap Resistive Plate Chambers and MicroMegas chambers which were constructed in collaboration with CERN detector experts.

EPS-HEP 2017, European Physical Society conference on High Energy Physics

5-12 July 2017

Venice, Italy

\footnotetext{
*Corresponding author

${ }^{\dagger}$ Speaker.
} 


\section{Introduction}

In 2014, Beamline for Schools (BL4S), an annual outreach program to interest high-school students for experimental particle physics, was initiated at CERN. Students from all over the world are invited to propose an experiment at the CERN Proton Synchrotron's T9 beamline using a mixed secondary beam (electrons, protons, pions, kaons, muons) with momenta of up to $10 \mathrm{GeV} / \mathrm{c}$ [1]. Two winning teams are invited to CERN to perform their experiment on site.

BL4S initially acquired a number of detectors which the students can use at their convenience. These include numerous $30 \times 20 \times 2 \mathrm{~cm}^{3}$ scintillator paddles, a $5 \mathrm{~mm}$ thick halo scintillator with a $3 \mathrm{~cm}$ opening, a calorimeter consisting of 16 lead-glass crystals obtained from the OPAL experiment [2] and two permanently installed $1 \mathrm{~m}$ long Threshold Cherenkov counters [3]. A $110 \times 110 \mathrm{~mm}^{2}$ 2D Delay Wire Chamber (DWC) with a resolution of $0.2 \mathrm{~mm}$ [4] is located at the entrance of the experimental zone with up to two more available for use in the experiment.

In the last years BL4S team decided to extend the selection of detectors in order to allow better particle identification and tracking. With the Threshold Cherenkov Counters a wide range of particle types and momenta can be distinguished. However, the maximum allowed pressure of $3 \mathrm{~atm}$ prohibits identifying protons in general and muons and pions below $2.5 \mathrm{GeV} / \mathrm{c}$. In this region a time-of-flight measurement can provide the necessary information. Therefore, three Multi-Gap Resistive Plate Chambers, known for and excellent timing resolution, were constructed.

Furthermore, BL4S offers students to build a spectrometer using a $0.5 \mathrm{Tm}$ dipole magnet in conjunction with a tracking detector. Initially, tracking was provided by DWCs which limited the spectrometer due to their size, readout and resolution. To extend the spectroscopy capabilities, four 1D MicroMegas detectors were constructed.

\section{Multi-Gap Resistive Plate Chambers}

The Multi-Gap Resistive Plate Chambers (MRPC) are gaseous detectors constructed from stacks of resistive glass plates with segmented anode and cathode electrodes placed as outermost layers. When charged particles ionise gas in gaps between glass plates, a large electric field causes avalanches of ionisation in each gap. The moving charge is picked up on readout strips and the timing of the charge deposition is read out on both sides of strips. The differential timing of signals reveals the position along strips and precise arrival times of particles, making MRPC detectors especially suitable for time-of-flight (TOF) measurements.

The design of BL4S chambers shown in Figure 1 is based on chambers used in the Time-OfFlight detector of the ALICE experiment [5]. Chambers have a surface area of $30 \times 30 \mathrm{~cm}^{2}$ with 24 strips. The gas filling mixture consists of $95 \% \mathrm{C}_{2} \mathrm{~F}_{4} \mathrm{H}_{2}$ and $5 \% \mathrm{SF}_{6}$ with a typical flow of $5 \mathrm{l} / \mathrm{h}$.

Two MRPCs were used for experiments in 2016 for particle identification with TOF method. Results are shown in Figure 1 (right). With a time-of-flight resolution of 400-500 ps and an effective flight distance of $5.36 \mathrm{~m}$, a clear distinction of signals from protons and other types of particles is visible. 

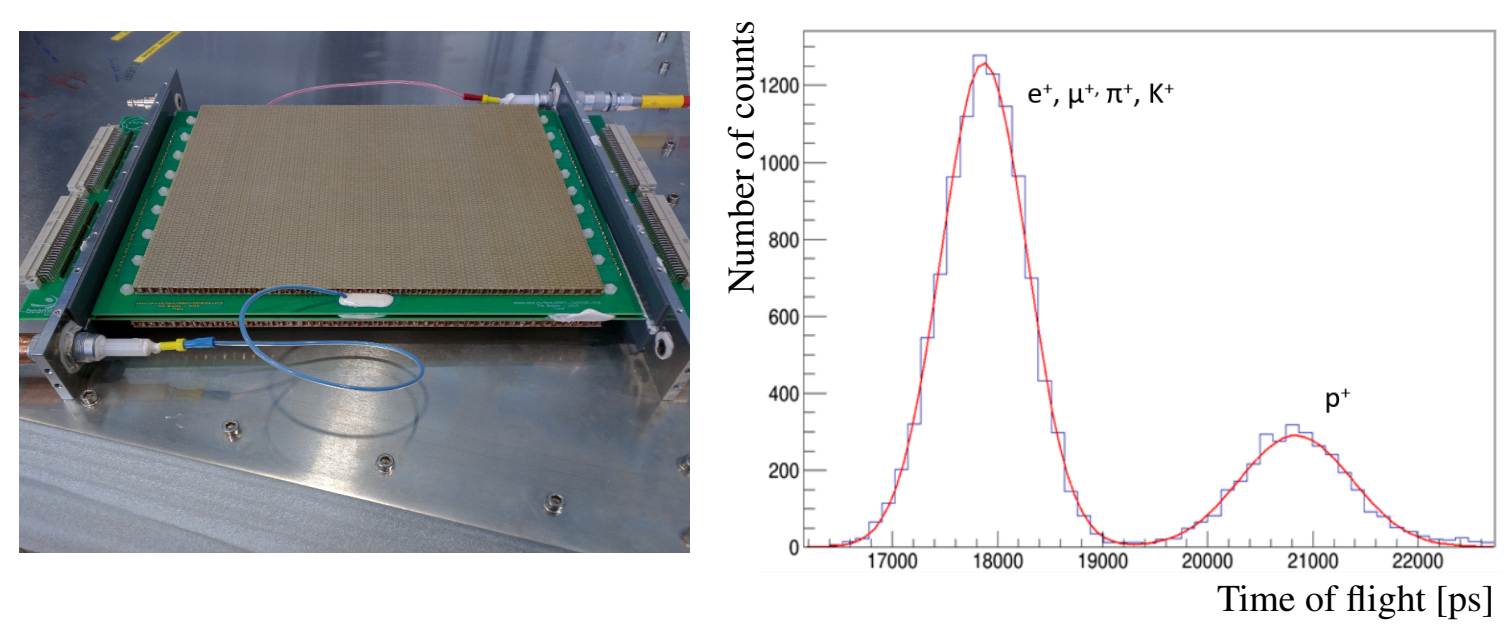

Figure 1: One of MRPCs constructed for BL4S (left) and results of time-of-flight measurement obtained using MRPCs during 2016 (right). The plot shows the time of flight of particles with momenta of $1.5 \mathrm{GeV} / \mathrm{c}$ in $\mathrm{T} 9$ for an effective flight distance of $5.36 \mathrm{~m}$.

\section{MicroMegas Tracking Detectors}

Micro Mesh Gaseous Structure (MicroMegas) detectors are finely granular one or twodimensional detectors which will be used in a large scale for the ATLAS New Small Wheel (NSW) upgrade project [6]. A typical layout of a MicroMegas chamber comprises a gas volume with an electric field spanned between a planar electrode and a segmented readout region. A finely pitched mesh separates the volume into a conversion and drift region with low electric field, and a thinner amplification region with high electric field. Thanks to this scheme a high detection efficiency combined with good spatial resolution is achieved.

In order to build a sensitive spectrometer and allow tracking of scattered particles, four $46 \times 46 \mathrm{~cm}^{2} 1 \mathrm{D}$ MicroMegas detectors have been developed. They follow closely the ATLAS NSW design and host 1024 readout strips with a pitch of $450 \mu \mathrm{m}$ and a resistive protection layer on top of the readout strips. The $5 \mathrm{~mm}$ (drift) and $128 \mu \mathrm{m}$ (amplification) thick gas volumes are separated by a stainless steel mesh with a granularity of 325 lines/inch, resting on pillars made of Pyralux. In contrast to classical designs, the mesh of the BL4S detectors is not fixed to the readout, thus it can be removed together with the drift electrode, easing construction and repairs. The chambers support a nominal electric field of up to $600 \mathrm{~V} / \mathrm{cm}$ in the drift region and $40 \mathrm{kV} / \mathrm{cm}$ for amplification in the $\mathrm{Ar}: \mathrm{CO}_{2}$ (93:7) gas mixture. Each chamber is read out by eight 128-channel APV-25 Front-End hybrids [7] and the RD-51 SRS system [8].

In total four chambers (one being shown in Fig. 2) have been built in the CERN EP-DT workshops and successfully tested at the CERN PS T9 beam line in an up to $10 \mathrm{GeV}$ mixed particle beam. Preliminary measurements showed a detection efficiency of over $97 \%$ leading to a release of the chambers for experiments in autumn 2017. So far, a pair of chambers was used by the BL4S finalists for monitoring the beam position as shown in Fig. 2. It facilitated the alignment of other detectors in beam and the observation of scattering of particles in air and an iron target. 

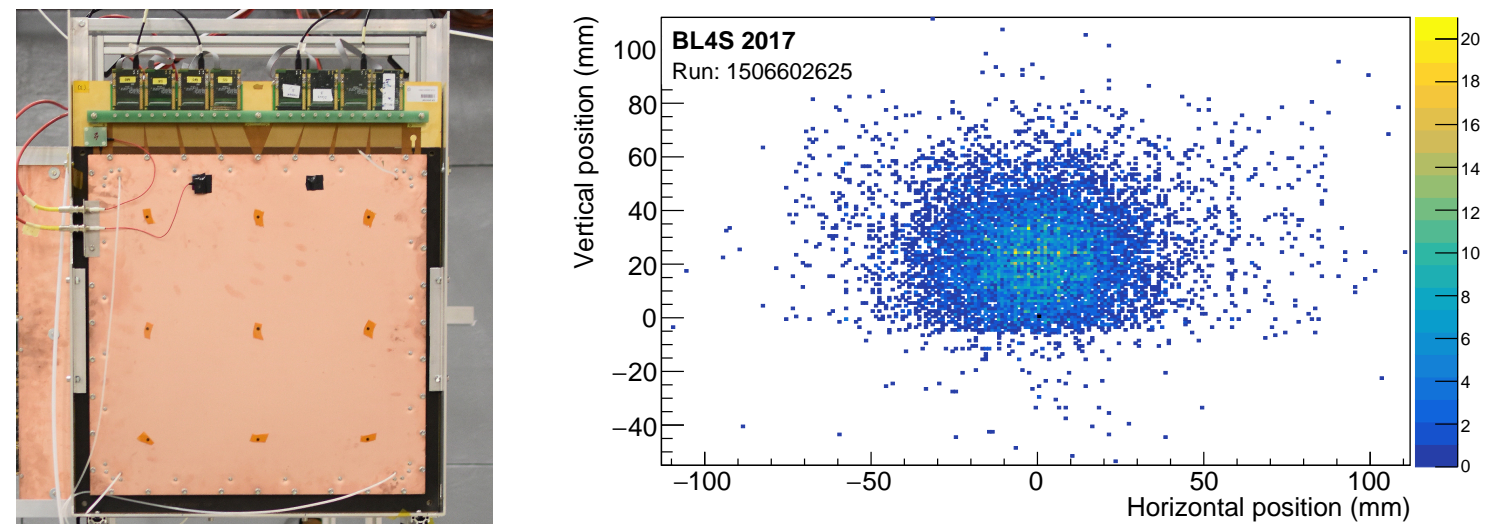

Figure 2: (left) A fully equipped BL4S MicroMegas detector. The long strip direction points upwards with 8 APVs on top of the chamber. (right) The beam spot at T9 as resolved by two BL4S MicroMegas chambers mounted at an angle of $90^{\circ}$ relative to each other.

\section{Conclusions}

Since its initiation in 2014, BL4S has been extending its selection of detectors to provide students with state of the art technology. Although a small sized core team does not allow extensive detector development, the availability of the CERN infrastructure and workshops enabled us to construct three MRPCs and four 1D MicroMegas chambers. The detectors were successfully integrated into experiments in 2016 and 2017 and judging by the submitted proposals are regularly considered by students in their experiment designs.

\section{Acknowledgements}

The construction and operation of the detectors would not have been possible without the generous help of many groups and individuals. We would like to thank members of the RD-51 collaboration, the CERN EP-DT PCB workshop and C. Williams and the MRPC team for the design and construction of the MRPC and MicroMegas chambers. Further thanks go to C. Joram, R. Dumps, L. Gatignon and the beamline physicists and technicians at T9 for their constant support of our data taking and its preparation.

\section{References}

[1] L. Durieu, O. Ferrando, J. Y. Hemery, J. P. Riunaud and B. Williams, The CERN PS east area in the LHC era, Conf. Proc. C970512 (1997) 228-230.

[2] OPAL collaboration, K. Ahmet et al., The OPAL detector at LEP, Nucl. Instrum. Meth. A305 (1991) 275-319.

[3] A. Bulte, Operation of Cerenkov Detectors at the PS East Hall, tech. rep., CERN, Dec, 1998. https://sba.web.cern.ch/sba/Documentations/Eastdocs/docs/Cerenkov.pdf. 
[4] A. Manarin and G. Vismara, The Delay Wire Chamber (DWC) Description, Tech. Rep. LEP/BI-TA/Note 85-3, CERN, Feb, 1985.

https://sba.web.cern.ch/sba/Documentations/Eastdocs/docs/DWC-Description.pdf.

[5] M. C. S. Williams, The Multigap RPC: The Time-of-flight detector for the ALICE experiment, Nucl. Instrum. Meth. A478 (2002) 183-186.

[6] ATLAS Collaboration, New Small Wheel Technical Design Report, Tech. Rep. CERN-LHCC-2013-006. ATLAS-TDR-020, Jun, 2013. https://cds.cern.ch/record/1552862.

[7] S. Martoiu et al., The APV25 0.25 $\mu \mathrm{m}$ CMOS readout chip for the CMS tracker, IEEE Nucl. Sci. Symp. Conf. 2 (2000) 9/113.

[8] S. Martoiu, H. Muller, A. Tarazona and J. Toledo, Development of the scalable readout system for micro-pattern gas detectors and other applications, JINST 8 (2013) C03015. 